\title{
ENGAGE! EMPOWER! ENCOURAGE!-Supporting Mundane Group Decisions on Tabletops
}

\author{
Mirko Fetter and Tom Gross \\ Human-Computer Interaction Group, University of Bamberg, Germany \\ mirko.fetter@uni-bamberg.de
}

\begin{abstract}
This paper presents an interaction model to support groups making decisions, aiming to ENGAGE the group in the interaction, EMPOWER all users to put forth their opinion, and ENCOURAGE the group to discuss the options. Based on the tabletop application MTEATSPLORE, we show how the interaction model helps to design a system to structure the decision making process and thus can lead to an effective and efficient yet inclusive support for mundane decisions.
\end{abstract}

Keywords: Group Decision Support, Interactive Tabletop, Multi-touch.

\section{Introduction}

Decision-making processes in organisations are getting more and more complex. The involvement of different stakeholders, highly specialised decision makers, as well as the increasing complexity of the decision environments, the high costs of subpar decisions and the factor time as a competitive resource, all lead to an increasing complexity when trying to find optimal decisions. Group Decision Support Systems (GDSS) are designed to help groups of people to reach consensus when looking for the best-fitting solution for an unstructured problem [3, 5]. They achieve this by inter alia structuring discussions, outlining procedures, and supporting the exploration of alternatives. GDSS are therefore applied in different settings from supporting merger and acquisition decisions of an executive board to helping a community find consensus on the best location for a new industrial estate.

GDSS are rarely applied in mundane group decision-making processes-that is, supporting those everyday, small decisions that are made in dyadic or polyadic settings of small groups (e.g., choosing a movie with friends; deciding on sights to visit on a family vacation; choosing a restaurant for a lunch with colleagues). While the actual costs of suboptimal decisions in these cases are not as high as in the first examples, still the perceived costs can lead to a poor movie experience, or a ruined vacation, maybe preceded by lengthy, exhausting and ineffective discussions.

In the following we introduce MTEATSPLORE (cf. Fig. 1), a system supporting small groups with the mundane decision of choosing a restaurant together. We layout the design rationale of MTEATSPLORE that is based on three phases (ENGAGE, EMPOWER and ENCOURAGE), which aim to structure the process of group decisions making, yet do not automate and patronise the final decision. We conclude with an outlook to future work. 


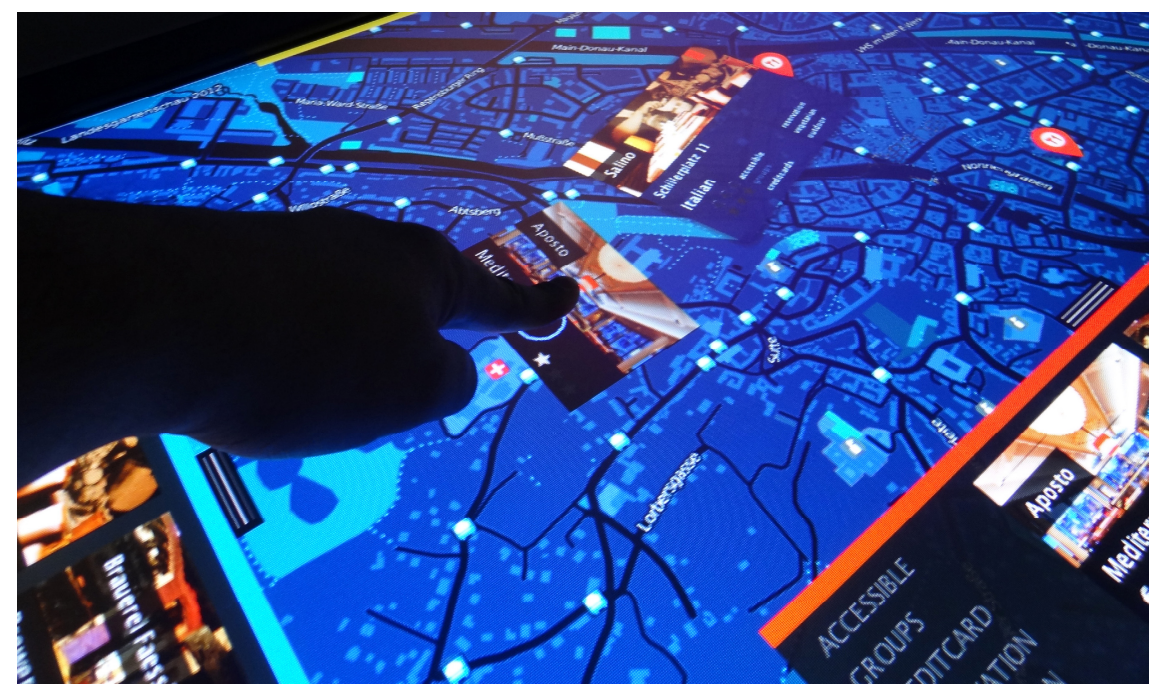

Fig. 1. Users interacting with the MTEATSPLORE on a multi-touch setup

\section{$2 \quad$ Related Work}

The diverse behavioural patterns of people when making decisions have been extensively researched for individuals as well as for groups [2, 9, 14]. A variety of studies assessed the consumer behaviour of groups in the marketing field [15] and shed light on what the critical factors are when small groups make mundane decisions. However, the number of systems currently available that support groups with making such mundane decisions is very low.

Business review sites-like Yelp ${ }^{1}$ for restaurants, tripadvisor ${ }^{2}$ for hotels and sightseeing, or $\mathrm{IMDB}^{3}$ for movies-allow users to extensively inform themselves about a product or service and so provide some help to form an opinion on the best available alternatives for mundane decisions like hotel booking or restaurant selection. But, such services do not offer convenient functionalities to enable groups to explore such information together, which would be a prerequisite for making a group decision. Even single users are not well supported throughout the decision making process, as besides filters and search functions mostly no additional tools to structure the decision making process are offered. There are no tools that allow to easily store a selection of promising alternatives, to rank or compare them directly.

While recommender systems [12] — which provide users with suggestions on the basis of their personal preferences-better support decision-making process as a whole, there are only few systems that provide recommendations for groups [1,7]. The group

\footnotetext{
${ }^{1}$ http: / / www . yelp.com

${ }^{2}$ http: //www.tripadvisor.com

${ }^{3}$ http: / / www. imab.com/
} 
recommender system CATS (Collaborative Advisory Travel System) [10] had a focus on designing an interaction concept for a group of people when collaboratively deciding for a skiing vacation. Further group recommenders supporting mundane group decisions are an extension to e-Tourism [4] to recommend tourist activities, as well as PolyLens [11] and AGReMo [1] for the selection of a movie in a group. However, those group recommender systems share two of the disadvantages that most recommender systems share: First, a high degree of automatisation, that at the end only provides one single option without further explanation, is often perceived by the users as to patronising [6]. Second, in order to be able to generate recommendations recommender systems need to know the preferences of the individual users upfront. This often requires an extensive phase of providing the system with the data (e.g. by rating a number of previously seen films). In ad-hoc scenarios, as we imagine them for MTEATSPLORE, such an approach is not feasible.

Finally, while there are already a number of more universal guidelines for developing interaction designs for applications on interactive tabletops, like, for example, the system guidelines for co-located, collaborative work on a tabletop displays by Scott et al., [13] the focus of our interaction model is on a higher level of specific applications-- that is, applications that support groups with making mundane decisions.

\section{Engage! EMPOWER! ECOURAGE!-A Model for Interaction}

The rationale behind using GDSSs in organisations are well documented, including phenomena like groupthink [8], where individual critical thinking is replaced by a thinking that leads to a preference of harmonic in-group consensus, although the decision made might be irrational from the own perspective. Yet, phenomena like the pressure to conform, individual domination, differing communication styles or differing group and individual goals also play a role when choosing a dining opportunity or a cinematic experience in a small group. For example, some people might find it difficult to put forward their own preferences out of politeness, shyness, or cautiousness. This might be especially true in combination with other group members that tend to be bolder, rash, or in other ways dominating. This might lead to suboptimal decisions, where some group members feel left behind or cause lengthy decisions. We, therefore, suggest systems that support groups with mundane decision-making.

By studying work done in the field of GDSS and the related area of group recommenders, we identified three basic requirements for successfully supporting mundane decision-making. First, such systems should make the process of reaching a decision more efficient and effective, yet should not constrain and automate the process, but ultimately leave the group in charge. Second, such systems should equalise member participation by giving a voice to each group member. And third, the application of such systems should be effortless and support a smooth, persuasive access. 
In order to address these requirements, we propose a three-phase model for the design of GDSS that support mundane decisions: ENGAGE, EMPOWER and ENCOURAGE.

ENGAGE-this first phase aims to engage the group in using the system. The goal is to persuade and invite the group into using the tool, although some of its members may be reluctant. We, therefore, suggest designing this phase in a way that it first proposes interaction possibilities for a single user. However, the goal is to design these first interactions of a facilitator or informal leader in a way they gradually draw in the other members of the group (e.g. by making the first user ask questions like "In which area to we want to eat?" or "At what time to we plan to go to the cinema?"). This phase is finished when all group members are interacting with the system.

EMPOWER - this second phase should be designed to equalise the users and to give all users a voice. In this phase all users are using the system. However, the focus in this phase is less on collaboration, but more on the individual exploring of alternatives, deciding on the preferred options based on the presented information, and putting forward the own preferences by actively choosing options. The design of this phase should optimally support structured individual work in order to boost the effectiveness and efficiency of the overall decision-making process. One goal in this phase is to not yet encourage to much discussion among the members, but at the most short chats like for example "This seems to be a nice option here...". When everybody feels informed and had the chance to put forth their preferences, this phase ends.

ENCOURAGE - the last phase aims to animate the group to have a conversation and to foster a discussion. Instead of presenting an automated decision based on the individual preferences, this phase should offer more freedom of decision but at the same time structure and guide the discussion of the members until they reach consensus. Therefore, the design should help to gradually reduce the options alongside the discussion process paving the way to the final decision and the end of the last phase.

In the following we describe the interaction concept of the MTEATSPLORE table based on theses three phases.

\section{MTEATSPLORE-Supporting Mundane Group Decision}

MTEATSPLORE is a multi multi-user, multi-touch tabletop application, designed to support a group of up to four users in finding a restaurant, which best fits the preferences of all group members. It is designed with various settings in mind including the placement of the tabletop in a hotel lobby, a tourist information office, or a conference centre. As one design goal was to empower participants that usually do not put there self forward in an unfamiliar setting, MTEATSPLORE is targeted towards ad-hoc formed, not well-acquainted groups of people (e.g., business partners, workshop participants at a conference). Additionally, it should satisfy the needs of more close-knit groups (e.g., colleagues, family members), with participants that 
know each other preferences better. In the following the interaction concepts and details on the design and implementation are outlined.

\subsection{The Interaction Concept of MTEATSPLORE}

The interaction design of MTEATSPLORE is based on the three phases ENGAGE, EMPOWER and ENCOURAGE and is depicted in Fig. 2.

Accordingly, the first phase (1) is designed to ENGAGE the group in the interaction with the table. We designed it to be operated by one user, who, by taking action motivates the group to use the table. This person can be seen as an informal leader or facilitator, for starting the decision process. By setting a location and a radius with one combined gesture (A) this user defines the area for which MTEATSPLORE suggests restaurants.

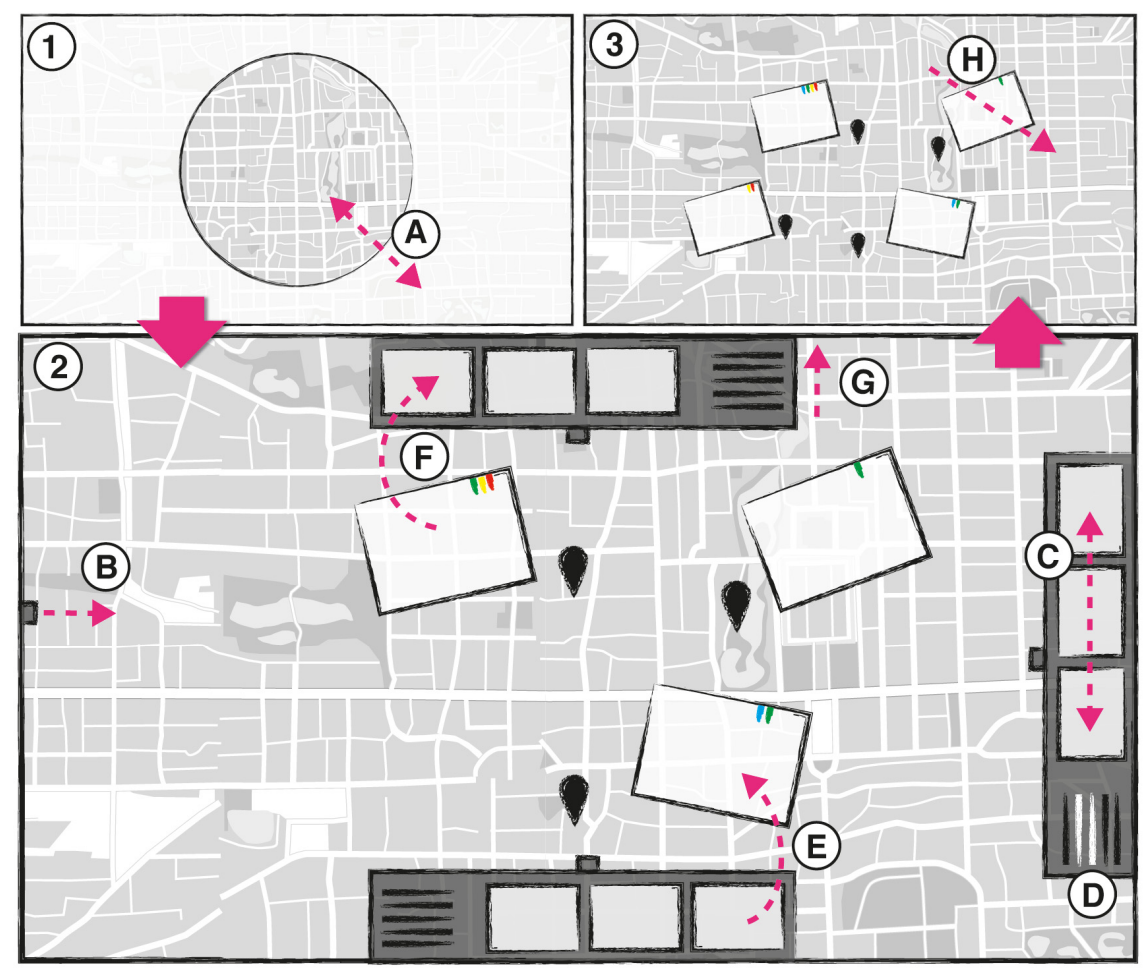

Fig. 2. Three phases of the MTEATSPLORE interaction concept presented counter clockwise representing the phases: 1) ENGAGE, 2) EMPOWER and 3) ENCOURAGE

This action leads to the next phase (2), which aims at EMPOWERing all users to freely and actively express their preferences by individually selecting restaurants they would like to visit. The group members place themselves at the four sides of the 
tabletop and drag out (B) their user workspaces. Each user now can individually go through the available restaurants with a swiping gesture (C). By activating filters (D) on the basis of personal preferences (e.g., only restaurant with outdoor dining options) the users can reduce the number of restaurants in their user workspace. If users find a restaurant interesting they can drag it (E) to the map in the centre-which serves as a shared workspace - to express this interest. When a restaurant sheet is dragged out, it offers more information, indicates the location on the map with a marker, and can be freely moved around in the shared workspace. Furthermore, a coloured marker in the colour of the users workspace indicates who is interested in this restaurant. If another user drags out the same restaurant, an animation visually merges the two restaurant sheets, and adds the coloured marker of this user. By dragging a restaurant sheet back in their workspaces (F) the users can reverse their choices. This action removes their coloured marker from the restaurant sheet if more users are interested in this restaurant, or respectively removes the restaurant sheet from the shared workspace, if the user was the only one who was interested in this restaurant. When users are done with the individual selection process, they can express this by closing their user workspace with a dragging gesture $(\mathrm{G})$.

When all user workspace are closed, the last phase (3) starts. This phase ENCOURAGEs an informed discussion among the users. While it would be technical feasible to only show the restaurants with most markers, our design explicitly does not have this sort of automatism. Like the first phase aims at smoothly starting the interaction, this phase wants to gently see the members off. By discussing the preferred restaurants based on the markers, the members can gradually reduce the number of restaurants removing them one by one with a strike-through gesture $(\mathrm{H})$ until the reach consensus with one restaurant left.

\subsection{Design and Implementation of MTEATSPLORE}

The aim of the interaction concept design of MTEatsplore was to transfer the interaction model ENGAGE, EMPOWER and ENCOURAGE to a concrete application design. It was developed in an iterative design process, which always was concerned with how good the interaction design supported the respective phase. The design process involved several prototyping stages from storyboarding, via paper mock-ups (cf. Fig. 3) and mid-fi design prototypes to an interactive wireframe prototype leading up to the final implementation. The final application is implemented in Java based on the Multitouch for Java Framework MT4 ${ }^{4}$, and makes usage of the TUIO protocol ${ }^{5}$ for receiving touch input. This combination allows the application to be run on a multitude of different commercial and non-commercial tabletop setups (e.g., the Samsung SUR40) on a variety of operating systems. For development and testing we used a low-cost setup consisting of a 40" LCD screen in combination with a Kinect camera and the software running on an 2,7 Ghz Quad-Core Intel Core i5 iMac with OS X 10.8.2. For the final system we used our custom tabletop consisting of a 1080p

\footnotetext{
${ }^{4}$ http : / / www . mt 4j . org

${ }^{5}$ http: //www.tuio.org/
} 
42" LCD screen and a combination PQ-Labs G4 42" Multi-touch Overlay with 32 simultaneous touch points and the software running on an $3,3 \mathrm{GHz}$ Intel Core i3 with Ubuntu 12.04 LTS.
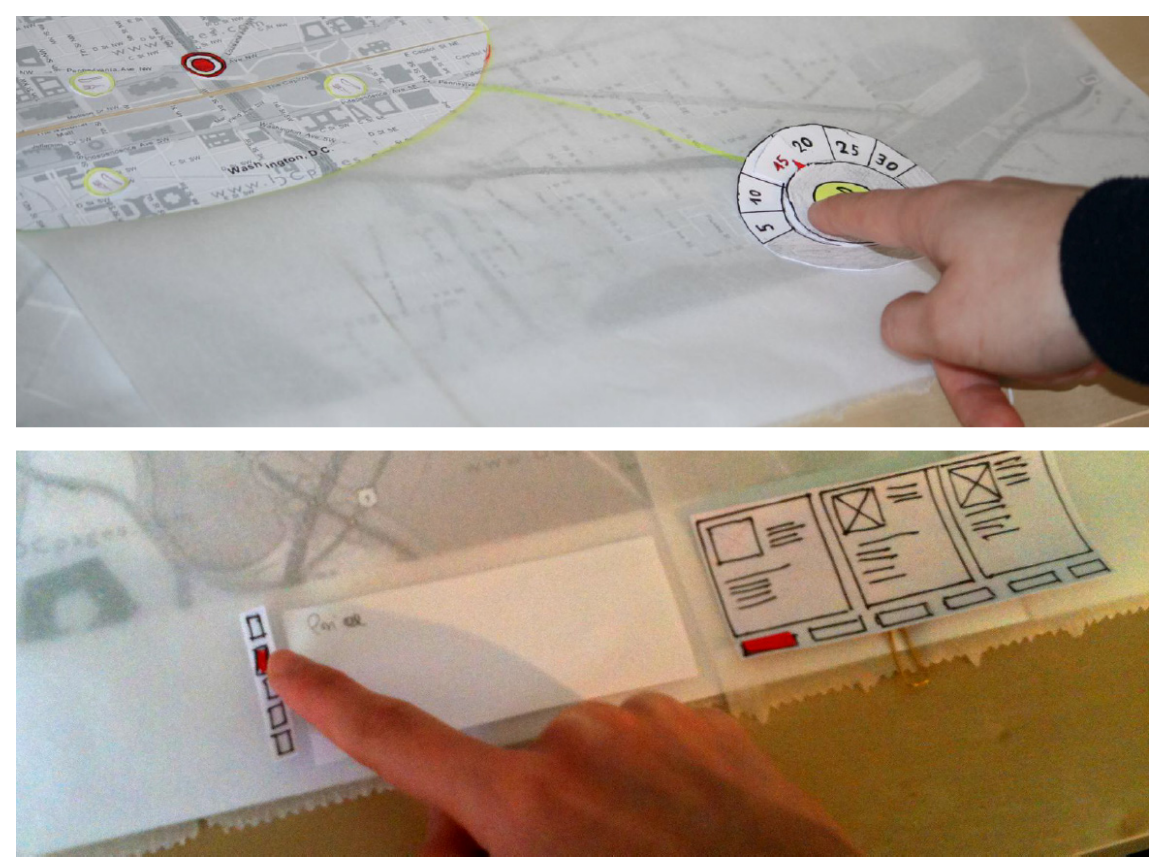

Fig. 3. Evaluation of paper prototypes of MTEATSPLORE: early design for the selection of the area in the first phase, and for applying filters in the second phase

\section{$5 \quad$ Conclusions and Future Work}

We presented MTEATSPLORE with three phases for supporting mundane group decision support: ENGAGE, EMPOWER and EnCOURAGE. How well MTEATSPLORE works, and whether the three phases will lead to better, more inclusive and more efficient decision making needs to be shown in a thorough evaluation. We plan to conduct studies that compare the performance and experience of groups choosing a restaurant together with and without MTEATSPLORE. Our aims are twofold. First, we are interested in how well MTEATSPLORE is able to convey the concept of ENGAGE, EMPOWER and ENCOURAGE, to trigger the intended behaviour, and to lead to the envisioned outcomes for each phase. Second, we are interested in how the concept supports the decision making process by analysing the satisfaction of the users with decision as well as with the decision process. Further observations of people using the table in the local tourist information will give additional insights.

Acknowledgments. We thank the members of the Cooperative Media Lab, especially Sascha Leicht and David Bimamisa. 


\section{References}

1. Beckmann, C., Gross, T.: AGReMo: Providing Ad-Hoc Groups with On-Demand Recommendations on Mobile Devices. In: European Conference on Cognitive Ergonomics, ECCE 2011, August 24-26, pp. 179-183. ACM Press, N.Y. (2011)

2. Castellan, N.J. (ed.): Individual and Group Decision Making: Current Issues. Lawrence Erlbaum, Hillsdale (1993)

3. DeSanctis, G., Gallupe, R.B.: A Foundation for the Study of Group Decision Support Systems. Management Science 33(5), 589-609 (1987)

4. Garcia, I., Sebastia, L., Onaindia, E., Guzman, C.: A Group Recommender System for Tourist Activities. In: Di Noia, T., Buccafurri, F. (eds.) EC-Web 2009. LNCS, vol. 5692, pp. 26-37. Springer, Heidelberg (2009)

5. Gray, P.: The Nature of Group Decision Support Systems. In: Burstein, F., Holsapple, C.W. (eds.) Handbook on Decision Support Systems 1, pp. 371-389. Springer, Heidelberg (2008)

6. Gross, T.: Supporting Effortless Coordination: 25 Years of Awareness Research. Computer Supported Cooperative Work: The Journal of Collaborative Computing and Work Practices 22(4-6), 425-474 (2013)

7. Jameson, A.: More Than the Sum of its Members: Challenges for Group Recommender Systems. In: Proceedings of the Working Conference on Advanced Visual Interfaces, AVI 2004, Gallipoli, Lecce, Italy, May 25-28, pp. 48-54. ACM Press, N.Y. (2004)

8. Janis, I.L.: Victims of Groupthink: A Psychological Study of Foreign-Policy Decisions and Fiascos. Houghton Mifflin, Boston (1972)

9. Janis, I.L., Mann, L.: Decision Making: A Psychological Analysis of Conflict, Choice, and Commitment. Free Press, N.Y. (1977)

10. McCarthy, K., Salamo, M., Coyle, L., McGinty, L., Smyth, B., Nixon, P.: Group Recommender Systems: A Critiquing Based Approach. In: Proceedings of the 11th International Conference on Intelligent User Interfaces, IUI 2006, Sydney, Australia, January 29-February 1, pp. 267-269. ACM Press, N.Y. (2006)

11. O’Connor, M., Cosley, D., Konstan, J.A., Riedl, J.: PolyLens: A Recommender System for Groups of Users. In: Proceedings of the Seventh European Conference on ComputerSupported Cooperative Work, ECSCW 2001, Bonn, Germany, September 16-20, pp. 199-218. Kluwer Academic Publishers, Dordrecht (2001)

12. Resnick, P., Varian, H.R.: Recommender Systems. Communications of the ACM 40(3), 56-58 (1997)

13. Scott, S.D., Grant, K.D., Mandryk, R.L.: System Guidelines for Co-Located, Collaborative Work on a Tabletop Display. In: Proceedings of the Eight European Conference on Computer-Supported Cooperative Work, ECSCW 2003, Helsinki, Finland, September 14-18, pp. 159-178. Kluwer Academic Publishers, Dortrecht (2003)

14. Tindale, R.S., Kameda, T., Hinsz, V.B.: Group Decision Making. In: Hogg, M., Cooper, J.M. (eds.) The SAGE Handbook of Social Psychology, pp. 381-406. SAGE Publications, London (2003)

15. Ward, J.C., Reingen, P.H.: Sociocognitive Analysis of Group Decision Making among Consumers. Journal of Consumer Research 17(3), 245-262 (1990) 